\title{
LA ECONOMÍA COLABORATIVA EN EL SECTOR DEL TRANSPORTE Y DEL ALOJAMIENTO: UNA APROXIMACIÓN DESDE EL DERECHO TRIBUTARIO*
}

\author{
THE COLLABORATIVE ECONOMY IN TRANSPORT AND ACCOMODATION \\ SECTOR. AN APPROXIMATION FROM TAX LAW
}

\author{
Genoveva Gil García**
}

\begin{abstract}
Resumen: En el presente trabajo se abordan los retos que la economía colaborativa representa para el Derecho Tributario. El desarrollo de las nuevas tecnologías ha favorecido la irrupción de nuevos modelos de negocio que, a través de plataformas digitales, han acrecentado los supuestos de consumo colaborativo hasta el punto de que ya podemos hablar de una economía colaborativa. El punto de partida del trabajo es el propio concepto de economía colaborativa, para después determinar los modelos de negocio concretos bajo el paraguas de aquélla. Seguidamente, se realiza un análisis, tanto desde la perspectiva de la fiscalidad directa como indirecta, en el sector del transporte y del alojamiento. Finalmente, el trabajo termina con las principales conclusiones alcanzadas.
\end{abstract}

Palabras clave: economía colaborativa, plataformas digitales, transporte, alojamiento, fiscalidad.

Abstract: The aim of this paper is to analyse the challenges posed by the collaborative economy to Tax Law. The development of new technologies has favoured the emergence of new business models which have increased the cases of collaborative economy. For this purpose, the author tackles first the concept of collaborative economy and the different business models. Then, the paper focuses on the direct and indirect taxation in the transport and accommodation service sector. Finally, the work explains the main conclusions reached.

Keywords: collaborative economy, collaborative platforms, transport, accommodation, taxation.

SUMARIO: I. INTRODUCCIÓN; II. LA ECONOMÍA COLABORATIVA: CONSIDERACIONES GENERALES; III. FISCALIDAD DIRECTA; 1. La tributación en el sector del transporte: las rentas obtenidas por el prestador del servicio y por la plataforma; 2. La tributación en el sector del alojamiento; 3. La tributación de las rentas percibidas por el prestador del servicio; 4. La tributación de las rentas obtenidas por la plataforma; IV. FISCALIDAD INDIRECTA; 1 . La tributación del prestador del servicio; 2. La tributación de la plataforma; V. CONCLUSIONES; VI. BIBLIOGRAFÍA.

* http://doi.org/10.15366/rjuam2019.39.004

Fecha de recepción: 31 de enero de 2019.

Fecha de aceptación: 11 de abril de 2019.

** Graduada en Derecho por la Universidad de Alicante. 


\section{INTRODUCCIÓN}

La economía colaborativa permite compartir bienes y servicios a través de plataformas de Internet y aplicaciones de las tecnologías de la información y la comunicación. Esta nueva realidad presenta grandes ventajas para una sociedad en la que los recursos infrautilizados pueden suponer una fuente de ingresos adicional, nada despreciable en el contexto actual de crisis económica. Ahora bien, esta nueva realidad plantea muchos interrogantes para el Derecho, los cuales han de ser resueltos para favorecer un adecuado desarrollo de lo que promete ser un importante motor para la economía.

En consecuencia, en el presente trabajo se realizará un análisis jurídico mediante el manejo de fuentes normativas, jurisprudenciales y doctrinales, con el objetivo de aportar cierta claridad a los retos a los que se enfrenta el Derecho Tributario en este punto. Asimismo, y aun centrándonos en el Derecho Tributario como disciplina autónoma, no se descuidarán los posibles aspectos de otras ramas del Derecho, como el Derecho del Trabajo, que puedan tener relevancia en el estudio del mismo.

Para ello, en primer lugar, se delimitará el concepto de economía colaborativa, así como los modelos de negocio y sujetos intervinientes, para seguidamente proceder a analizar la tributación de las distintas manifestaciones de capacidad económica, tanto desde el punto de vista de la fiscalidad directa como de la fiscalidad indirecta. En este punto, se ha de tener en cuenta que únicamente se tratarán los sectores del transporte y del alojamiento, pese a que son numerosos los ámbitos en los que la economía colaborativa tiene cabida.

En el apartado relativo a la fiscalidad directa se abordará la calificación de las distintas rentas obtenidas por los actores con una estructura separada por sectores, sin pasar inadvertidos ciertos aspectos de relevancia para la fiscalidad internacional. Por último, para el estudio de la fiscalidad indirecta distinguiremos los ingresos obtenidos en función del sujeto pasivo.

Es nuestra intención, por último, finalizar este trabajo con la realización de algunas conclusiones, en las que vuelve a ponerse de manifiesto la importancia que tiene en la actualidad la digitalización de la economía y la economía colaborativa por sus implicaciones en el ámbito del Derecho Tributario.

\section{LA ECONOMÍA COLABORATIVA: CONSIDERACIONES GENERALES}

El consumo colaborativo parte de la existencia de recursos infrautilizados y de personas necesitadas de los mismos, y por ello surgen iniciativas voluntarias entre los miembros de la comunidad tendentes a vender, compartir, prestar, alquilar, regalar o intercambiar bienes e incluso servicios ${ }^{1}$. No se trata de un fenómeno novedoso, si bien con el desarrollo

\footnotetext{
ANTÓN ANTÓN, Á. y BILBAO ESTRADA, I., «El consumo colaborativo en la era digital: un nuevo reto para la fiscalidad», en Documento de Trabajo, IEF, núm. 26, 2016, p. 7.
} 
de las nuevas tecnologías y la aparición de las TIC, ha proliferado hasta el punto de que podemos afirmar la existencia de una economía colaborativa.

Así, en los últimos años debido a la crisis económica, a la necesidad de empleo, y con ello, la tendencia de la sociedad al ahorro, aprovechamiento y reutilización de los recursos, estamos asistiendo a un refinamiento y consolidación de la llamada economía colaborativa. Ello se debe a la irrupción de un tercer actor en las relaciones tradicionales entre prestador y usuario: las plataformas digitales.

La economía colaborativa puede resultar muy beneficiosa pues crea nuevas oportunidades para consumidores y emprendedores. Tal y como ha puesto de manifiesto la Comisión Europea ${ }^{2}$, puede contribuir de manera importante al empleo y al crecimiento en la Unión Europea (en adelante, UE) si se fomenta y desarrolla de manera responsable. Por un lado, en favor de los emprendedores, ofrece nuevas modalidades de empleo, así como trabajos flexibles y nuevas fuentes de ingresos. Por otro lado, en favor de los consumidores, amplía la oferta de productos y servicios y procura precios más bajos. Además, promueve un mejor reparto de los activos y un uso más eficaz de los recursos, lo que puede favorecer la protección del medio ambiente y un avance en la transición hacia la economía circular.

Ahora bien, aunque el potencial económico y social de esta nueva realidad es claro ${ }^{3}$, al mismo tiempo genera un elevado grado de incertidumbre en relación con los derechos y obligaciones de los intervinientes en la misma, siendo esta una circunstancia que puede disuadir a las personas de participar en la economía colaborativa e impedir su desarrollo. De hecho, según una encuesta Eurobarómetro sobre el uso de las plataformas colaborativas, un $52 \%$ de los ciudadanos de la UE conoce los servicios ofrecidos por la economía colaborativa y tan solo un $17 \%$ ha utilizado estos servicios al menos una vez ${ }^{4}$. Estos datos evidencian la necesidad de que los Estados realicen esfuerzos regulatorios que aporten seguridad jurídica y contribuyan al impulso de los nuevos modelos de negocio.

Los interrogantes surgen tanto para el Derecho Laboral, Administrativo, Mercantil, Civil como para el Derecho Tributario, y será necesaria la simbiosis entre todos ellos para dar una respuesta que aporte confianza a los usuarios. Así, surgen problemas en relación con la precariedad laboral; determinar si el trabajador lo es por cuenta propia o por cuenta ajena, con las consecuentes implicaciones en materia de Seguridad Social; la protección del consumidor frente a prácticas desleales y, en general, la tutela de sus derechos; la pri-

Comunicación de la Comisión de 2/06/2016: «Una Agenda Europea para la economía colaborativa», p. 2.

Se estima que en el futuro la economía colaborativa puede suponer unos ingresos de entre $160 \mathrm{y}$ 572 miles de millones de euros en toda la UE (Opinion of the European Economic and Social Committee, 6/11/2017: «Taxation of the collaborative economy, analysis of possible tax policies faced with the growth of the collaborative economy», p. 4).

4 Commission Staff Working Document 2/06/2016, accompanying the document: Communication from the Commission: «A European Agenda for the collaborative economy - supporting analysis», p. 5. 
vacidad y el tratamiento de los datos personales ${ }^{5}$; la competencia desleal para los actores tradicionales del mercado; el empleo de monedas virtuales y sociales como instrumentos operativos de esta economía; y especialmente en relación con la tributación. En concreto, en el ámbito tributario se suscitan dificultades en cuanto a la identificación de los contribuyentes y los ingresos sometidos a imposición, la falta de información sobre los prestadores de servicios, una planificación fiscal «agresiva» en el sector digital, el intercambio insuficiente de información y las diferencias fiscales en la UE.

El problema común a todas estas cuestiones y sobre el que se centran los debates actuales, es la propia delimitación del concepto de economía colaborativa, esto es, la identificación de las distintas modalidades de esta economía, así como de los sujetos intervinientes. A los efectos de este trabajo, nos acogemos en relación con esta cuestión a las orientaciones dadas por las distintas instituciones y organismos de la UE.

De acuerdo con la Comisión Europea, la economía colaborativa se refiere a «modelos de negocio en los que se facilitan actividades mediante plataformas colaborativas que crean un mercado abierto para el uso temporal de mercancías o servicios ofrecidos a menudo por particulares» ${ }^{6}$. Las transacciones de la economía colaborativa no suelen implicar un cambio de propiedad y pueden llevarse a cabo con o sin ánimo de lucro. En ella participan tres categorías de agentes: i) prestadores de servicios que comparten activos, recursos, tiempo y/o competencias (pueden ser particulares que ofrecen servicios de manera ocasional o prestadores de servicios que actúen a título profesional ${ }^{7}$ ); ii) usuarios de dichos servicios; iii) intermediarios (plataformas colaborativas), que conectan a los prestadores con los usuarios $\mathrm{y}$ facilitan las transacciones entre ellos ${ }^{8}$.

Por lo que se refiere a los modelos de negocio, el propio Comité Económico y Social Europeo (en adelante, CESE), considera que el verdadero desafío consiste en delimitar las distintas modalidades de esta economía, para así proponer enfoques reguladores diferencia-

\footnotetext{
5 Desde el 25 de mayo de 2018 es aplicable el nuevo Reglamento general de protección de datos (UE) 2016/679.

6 Comunicación de la Comisión de 2/06/2016, ob. cit., p. 3.

7 Cada vez es más frecuente oír hablar del término «prosumidor», acuñado por Alvin Toffler en 1980, y entendido como «figura ecléctica que aúna las funciones asociadas a la figura del productor y del consumidor». JARNE MUÑOZ, P., «El prosumidor como figura clave en el desarrollo del Derecho del Consumo derivado del mercado digital», en Revista CESCO de Derecho de Consumo, núm. 19, 2016, p. 43.

8 Es conveniente distinguir la economía colaborativa de otros negocios digitales que pueden tener características similares, pero que como Lucas Durán opina, no deberían considerarse incluidos en tal definición. «Así, cuando un portal de internet gestiona reservas de hotel (v.gr. Booking.com), o presta servicios de música o visionado de películas a través de la red (v.gr. Spotify o Netflix) o, simplemente, vende libros electrónicos o bienes diversos (v.gr. Amazon), no puede decirse que, estrictamente, nos encontremos en el ámbito de la economía colaborativa, sino únicamente ante un nuevo canal de distribución (on-line) propio de la revolución tecnológica que hemos vivido en los últimos tiempos». LUCAS DURÁN, M., «Problemática jurídica de la economía colaborativa: especial referencia a la fiscalidad de las plataformas», en Anuario de Facultad de Derecho (Universidad de Alcalá), núm. 10, 2017, p. 135.
} 
$\operatorname{dos}^{9}$. Menciona, por ejemplo, la «economía bajo demanda», en la que se atomiza la fuerza de trabajo creando más flexibilidad, pero aumentando el riesgo de precarización laboral; o la gift economy o «economía de regalo» en la que se comparten bienes y servicios de manera altruista. Ahora bien, en este trabajo nos sumamos a la propuesta de clasificación de las plataformas realizada por Montesinos Oltra ${ }^{10}$. Si bien partiendo de la propuesta realizada por este autor, consideramos necesaria la introducción de una tercera categoría de modelo de negocio perfectamente diferenciable de las anteriores. Esta distinción será de gran relevancia para la determinación de su tratamiento fiscal.

Así pues, de acuerdo con dicho autor, encontramos, en primer lugar, aquellas plataformas que podríamos denominar de «filosofía colaborativa», en las que el modelo de negocio consiste: por un lado, en la relación colaborativa entre personas físicas que no entraña contraprestación; $y$, por otro lado, en el intercambio directo o indirecto de bienes o servicios, en las que no es posible hablar de un ánimo de liberalidad. En esta categoría podemos incluir plataformas como los bancos del tiempo, de intercambios de comida (v.gr. Share Your Meal), de intercambios de casa (v.gr. Lovehomeswap o Guest to Guest) o BlaBlaCar ${ }^{11}$.

En segundo lugar, tenemos las plataformas que conectan a personas oferentes de bienes o servicios, y a demandantes dispuestos a satisfacer por ellos una contraprestación dineraria. Este grupo es el que cuenta con una mayor relevancia económica, por lo que su importancia tributaria es indiscutible. Por ejemplo, encajarían en esta definición Airbnb ${ }^{12}$, Wallapop, Letmespace, Wimdu, 9flats.com, Handiscover, Drivy....etc.

9 Dictamen del Comité Económico y Social Europeo sobre la «Comunicación de la Comisión al Parlamento Europeo, al Consejo, al Comité Económico y Social Europeo y al Comité de las Regiones - Una Agenda Europea para la economía colaborativa», 521. ${ }^{\circ}$ Pleno del CESE de los días 14 y 15 de diciembre de 2016 , p. 34.

10 MONTESINOS OLTRA, S., «Los actores de la economía colaborativa desde el punto de vista del Derecho Tributario», en Revista Economía Industrial, núm. 402, 2016, pp. 50-51.

11 Se trata de una plataforma fundada en Francia en 2006, que conecta a conductores que viajan de una ciudad a otra con asientos vacíos, con aquellas personas que pretenden viajar al mismo destino. Sobre esta plataforma se hablará más adelante con ocasión de la Sentencia del Juzgado de lo Mercantil de Madrid, núm. 30/2017 de 2 de febrero.

12 Se trata de una plataforma fundada en 2008 en Estados Unidos, que permite a los usuarios alquilar las habitaciones vacías o bien su casa por completo. En concreto, sus creadores se inspiraron en su propia experiencia, ya que subarrendaron su vivienda ofreciendo dormir en colchones inflables (de ahí el nombre Airbnb: Airbed \& Breakfast).

Aunque en nuestro trabajo hemos considerado a la plataforma Airbnb incluida en el segundo modelo de negocio, diversos estudios muestran que «un porcentaje relevante de las relaciones económicas que se producen bajo el paraguas de Airbnb no constituyen lo que podrá denominarse «economía colaborativa» sino un negocio mobiliario más tradicional». SANZ GÓMEZ, R., «Airbnb, ¿economía colaborativa o economía sumergida? Reflexiones sobre el papel de las plataformas de intermediación en la aplicación de los tributos», en Documento de Trabajo IEF, núm. 15, 2017, p. 65. 
Por último, proponemos una tercera categoría, en la que habríamos de situar a aquellas plataformas que no se dedican a prestar un servicio de la sociedad de la información ${ }^{13}$, sino que prestan el servicio subyacente, y que consideramos puede resultar dudosa su adscripción a la economía colaborativa. En este sentido la Sentencia del Tribunal de Justicia de la Unión Europea (TJUE) de 20 de diciembre de 2017 (caso Asociación Profesional Élite Taxi contra Uber Systems Spain, S.L), ha despejado varios interrogantes en lo que se refiere a la calificación de las relaciones que surgen en el seno de la economía colaborativa. Tanto es así, que la plataforma Uber ha sido calificada por el TJUE como una empresa de transporte, que, si bien emplea a conductores no profesionales, no es una plataforma digital de intermediación ${ }^{14}$. No obstante, no hemos de olvidar que lo determinado por esta Sentencia tan solo es aplicable a la plataforma Uber, aunque no podemos descartar que en el futuro se resuelva en un mismo sentido en relación con la calificación de otras plataformas. En este sentido, el CESE recomienda la creación de una agencia independiente de calificación europea de las plataformas digitales.

Cada uno de estos modelos puede incluir sectores muy variados (en el tercero, por el momento, solo ubicaríamos con certeza a una plataforma concreta), desde transporte, alojamiento o servicios para el hogar, hasta financiación (crowdfunding ${ }^{15}$ ), educación o alimentación. Sin embargo, nos centraremos en el estudio de los dos primeros por encontrarse entre los cinco sectores clave de la economía colaborativa y por presentar los datos más elevados en porcentaje de ingresos netos $(31.9 \%$ y $27.3 \%)$ y en porcentaje de comercio $(53.7 \%$ y $12.4 \%)$ en $2015^{16}$.

Distinguidos los tres modelos de negocio sobre los que se basa nuestro trabajo, las relaciones que pueden surgir en los mismos permiten múltiples combinaciones. Así, podemos hablar de relaciones o transacciones de particular a particular (peer-to-peer, $P 2 P$ ), de empresario a particular (business to consumer, B2C), de particular a empresario $(C 2 B)$ y de empresario a empresario $(B 2 B)$. Es decir, relaciones en las que la calificación del prestador del servicio y/o del usuario puede ser de particular y/o de empresario. Será determinante para su tratamiento jurídico saber de cuál de ellas se trata, así como, igualmente, evaluar por separado las relaciones plataforma y usuario, plataforma y prestador de servicios, y prestador de servicios y usuario, teniendo en cuenta que la plataforma, por lo general, será

13 Conforme al art. 1.2 de la Directiva 98/34, al que remite el art. 2 a) de la Directiva 2000/31, se entiende por servicio de la sociedad de la información «todo servicio prestado normalmente a cambio de una remuneración, a distancia, por vía electrónica y a petición individual de un destinatario de servicios». La transposición de esta Directiva se ha realizado en la Ley 34/2002, de 11 de julio, de servicios de la sociedad de la información y de comercio electrónico.

14 Esta Sentencia se abordará más adelante, con ocasión del tratamiento de la fiscalidad directa en la economía colaborativa.

15 En este sentido puede verse, MARTÍNEZ MUÑOZ, Y., «El tratamiento fiscal del crowdfunding», en Revista Quincena Fiscal, núm. 14, 2015.

16 Commission Staff Working Document 2/06/2016, accompanying the document: Communication from the Commission, ob.cit., pp. 8-9. 
de titularidad de una persona jurídica, sin perjuicio de que sea posible la titularidad por parte de una persona física.

Tras determinar los distintos modelos de negocio de la economía colaborativa, así como los sujetos intervinientes, procederemos a analizar la tributación de las distintas manifestaciones de riqueza que puedan surgir en la economía colaborativa, tanto desde el punto de vista de la fiscalidad directa como indirecta. En este sentido, consideramos, siguiendo a Antón Antón ${ }^{17}$, que no deben crearse figuras impositivas específicas, sino que las figuras existentes han de adaptarse a las nuevas realidades. Ahora bien, los problemas y cuestiones que van a surgir en materia tributaria son múltiples, y habrán de estudiarse caso por caso en función del modelo de negocio y sector de que se trate. Por esta razón, como ya se adelantó, delimitamos el objeto de estudio de este trabajo centrándonos en los sectores del alojamiento y transporte, tomando como referencia, principalmente, a las plataformas Uber, Blablacar, Airbnb y Lovehomeswap.

\section{FISCALIDAD DIRECTA}

\section{La tributación en el sector del transporte: las rentas obtenidas por el prestador del servicio y por la plataforma}

Para abordar el estudio de la fiscalidad directa en el sector del transporte, hemos de tener en cuenta que el principal problema reside, por un lado, en determinar si los servicios son ofrecidos por prestadores profesionales o por particulares de manera ocasional, $\mathrm{y}$, por otro lado, en determinar si la plataforma presta un servicio de la sociedad de la información o el servicio subyacente.

En relación con la primera problemática, es necesario concretar la intención de los prestadores del servicio de transporte, que puede consistir, o bien en la obtención de rentas, o bien en la compartición de gastos. En este sentido, la Comisión Europea recoge los factores más importantes que deben darse en una prestación de servicios entre pares, para que el prestador del servicio subyacente pueda considerarse un comerciante o prestador profesional, si bien, especifica que «ninguno de ellos sería por sí mismo suficiente» ${ }^{18}$. Así pues, considera que los mencionados factores son la frecuencia de los servicios, considerando que una mayor frecuencia en la prestación del servicio podría indicar que está actuando con un propósito relacionado con su actividad económica; el fin lucrativo, indicando que es probable que los prestadores que obtienen una remuneración superior a la compensación de los costes persigan tal fin; $y$, el nivel de volumen de negocio.

\footnotetext{
17 ANTÓN ANTÓN, Á., «Implicaciones fiscales de la economía digital en la era digital», en MORENO GONZÁLEZ, S. (dir.) y GÓMEZ REQUENA, J.A. (ed.), Tendencias y desafios fiscales de la economía digital, (Aranzadi), 2017, p. 565.

18 Comunicación de la Comisión de 2/06/2016, ob. cit., pp. 10-11.
} 
De este modo, como resultado de la calificación que nos merezca en el caso concreto la prestación del servicio, si apreciamos que el sujeto es un comerciante o prestador profesional, las rentas que obtenga del ejercicio de su actividad estarán sujetas al IRPF por tratarse de un rendimiento de actividades económicas con base en el art. 27.1 de la Ley 36/2006 del Impuesto sobre la Renta de las Personas Físicas (en adelante, LIRPF). En cambio, si consideramos que el prestador del servicio es un particular cuyo interés se limita a la estricta compartición de gastos, en nuestra opinión estaríamos ante un supuesto de no sujeción al citado impuesto.

Ahora bien, también es frecuente que los particulares además de minorar los costes relacionados con el vehículo obtengan ocasionalmente ciertos ingresos. En consecuencia, algunos autores a los que nos sumamos ${ }^{19}$, proponen la fijación de una exención o de un supuesto de no sujeción hasta un cierto umbral por la posible renta obtenida en esta modalidad de consumo; es decir, cuando los particulares no se limiten a exigir una mera compartición de $\operatorname{gastos}^{20}$. De hecho, algunos países de nuestro entorno, como Francia, parecen decantarse por esta opción que, además, permitiría diferenciar de una manera objetiva entre los comerciantes y los particulares que prestan el servicio de manera ocasional. Así, el Senado francés, en una propuesta de 29 de marzo de $2017^{21}$, estableció un umbral de $3.000 €$ anuales por debajo del cual los sujetos estarían exentos, pues se considerarían prestadores de servicios ocasionales ${ }^{22}$.

En este sentido, la finalidad de la plataforma Blablacar (ejemplo del primer modelo de negocio) es que los usuarios hagan un uso compartido de los vehículos (carpooling o covoiturage) y, además, tal y como establece la propia plataforma en sus «Condiciones Generales de Uso», el prestador del servicio («conductor») no puede solicitar una cantidad de costes compartidos superior a los costes reales del trayecto, los cuales puedan generarle un beneficio. De hecho, la plataforma se reserva el derecho a suspender la cuenta de quien «utilice un vehículo de alquiler con conductor u otro vehículo comercial, taxi o vehículo de empresa con el objetivo de generar un beneficio a través de la plataforma», o también

19 Por ejemplo, ANTÓN ANTÓN, Á. y BILBAO ESTRADA, I.: «El consumo colaborativo en la era digital: un nuevo reto para la fiscalidad», ob. cit., p. 22.

20 En este sentido, Machancoses García considera que urge una interpretación sobre la calificación a efectos del IRPF de los supuestos en los que la prestación del servicio se ha realizado ocasionalmente y los ingresos son nimios. No obstante, le parecería «desproporcionado calificar como rendimientos de actividades económicas, o ganancias patrimoniales, los ingresos ocasionales y de escaso valor económico que puede obtener un particular por la realización de un servicio de transporte en escasas ocasiones puntuales, máxime en un contexto de crisis económica, en el que estos ingresos pueden ser un desahogo para muchos particulares». MACHANCOSES GARCÍA, E., «Economía de plataforma en los servicios de transporte terrestre de pasajeros: Retos tributarios de la imposición directa sobre el usuario y la plataforma», en Revista Quincena Fiscal, núm.15, 2017, p. 57.

${ }_{21}$ La mencionada propuesta se encuentra disponible en: $<$ http://www.senat.fr/rap/r16-481-2/r16-481-20. $h t m l>$ [Consultado el 18/06/2019].

22 En realidad, estas propuestas de los Estados de establecimiento de umbrales, no solo se prevén para el sector del transporte, sino para todos aquellos sectores en los que se plantea el problema de distinguir entre la prestación de servicios profesionales y la prestación de servicios ocasionales (comerciante vs. particular). 
en el caso de que cualquier otro motivo sugiera a Blablacar que se está utilizando la plataforma para obtener un beneficio ${ }^{23}$. Por lo tanto, en principio, las prestaciones de servicio que se produzcan en el seno de esta concreta plataforma no van a generar riqueza para sus usuarios; siendo el resultado, a nuestro entender, la no sujeción de tales operaciones. No obstante, en el caso de que el conductor obtuviera algún tipo de beneficio deberían entrar en juego las propuestas de los umbrales de no sujeción y exención. Para este fin, sería útil la colaboración de la plataforma con la Administración Tributaria a los efectos de obtener información suficiente por parte de las autoridades fiscales.

Por lo que se refiere a la segunda cuestión planteada, como sucede en el anterior supuesto, la Comisión Europea establece una serie de criterios para determinar si una plataforma colaborativa ofrece además del servicio de la sociedad de la información, el servicio subyacente. Así, entre otros, determina que si la plataforma impone el precio final que debe pagar el usuario, fija otras condiciones contractuales clave, o si existe una relación laboral entre la plataforma y la persona que presta el servicio subyacente, existen indicios de que la plataforma ejerce una influencia o control sobre el prestador del servicio subyacente, de modo que se puede considerar que la plataforma presta a su vez el servicio en cuestión.

Esto será determinante para calificar la relación existente entre las plataformas y sus colaboradores o proveedores, lo cual es de especial relevancia desde el punto de vista del Derecho del Trabajo ${ }^{24}$ y, en consecuencia, para el Derecho Tributario.

En este sentido, la STJUE de 20 de diciembre de 2017, ha arrojado mucha claridad en relación con esta problemática. En concreto, el TJUE ha zanjado la cuestión de si Uber debe considerarse una actividad de transporte o un servicio electrónico de intermediación. Tal y como adelantamos, el Tribunal ha concluido que Uber «no se limita a un servicio de intermediación consistente en conectar, mediante una aplicación para teléfonos inteligentes, a un conductor no profesional que utiliza su propio vehículo con una persona que desea realizar un desplazamiento urbano», sino que también «establece al menos el precio máximo de la carrera, que recibe este precio del cliente para después abonar una parte al conductor no profesional del vehículo y que ejerce cierto control sobre la calidad de los vehículos, así como sobre la idoneidad y el comportamiento de los conductores, lo que en su caso puede entrañar la exclusión de estos». Así, concluye que el servicio de intermediación que realiza Uber forma parte de un servicio global cuyo elemento principal es un servicio de transporte, y que no responde a un servicio de la sociedad de la información.

De este modo, Uber es una plataforma que presta, además de un servicio de intermediación, un servicio de transporte. En consecuencia, se puede concluir que entre el prestador

23 Disponible en: <https://www.blablacar.es/conoce-blablacar/condiciones-generales-de-uso $>$ [Consultado el 18/06/2019].

24 Tal relevancia puede verse, por ejemplo, en HERNÁNDEZ BEJARANO, M., «El apoyo europeo al modelo de economía colaborativa: algunas cuestiones y propuestas para afrontar una regulación laboral y de Seguridad Social», en Revista Española de Derecho del Trabajo, núm. 192, 2016, pp. 174-181. 
del servicio subyacente y la plataforma existe una relación laboral, ya que se implica activamente con los proveedores del servicio. Esto determina que las rentas obtenidas por el primero estén sujetas al IRPF por tratarse de un rendimiento del trabajo (art. 17.1 LIRPF), y por ello, Uber adquiere la obligación formal de practicar las retenciones correspondientes, conforme al art. 99.2 LIRPF.

Por el contrario, la plataforma Blablacar, de acuerdo con la Sentencia del Juzgado de lo Mercantil de Madrid núm. 30/2017 de 2 de febrero, presta un servicio de la sociedad de la información y no un servicio de transporte. Así, el referido pronunciamiento señala que «Blablacar ha generado una plataforma no para organizar el transporte, sino para poner en contacto a particulares que quieren realizar un viaje juntos, y compartir determinados gastos del trayecto». Los conductores «no están contratados por Blablacar, ni pertenecen a una empresa o a una industria dedicada a este fin». Esto refuerza nuestra tesis de que en este modelo de negocio los conductores no obtienen rendimientos del trabajo y, en todo caso, podría entenderse que, si se superase un determinado umbral de ingresos, cabría hablar de rendimientos de actividades económicas. De este modo, no superado el umbral establecido, el conductor no resultaría efectivamente gravado, por tratarse de un ingreso ocasional. En el concreto caso que nos ocupa, dadas las condiciones establecidas por la plataforma Blablacar, no será frecuente la obtención de beneficios, sino una mera compartición de gastos.

Una vez calificados los rendimientos que los prestadores de servicios pueden obtener en este sector de la economía colaborativa, nos referimos seguidamente a los usuarios y a las plataformas digitales que intervienen asimismo en esta relación triangular, teniendo en cuenta que los primeros no obtienen ningún rendimiento por su participación en este sector, pues únicamente reciben el servicio.

La fiscalidad de las rentas obtenidas por la plataforma, en principio, no presenta dificultades, pues los rendimientos obtenidos como consecuencia del ejercicio de su actividad económica, ya sea la prestación de un servicio de intermediación o el propio servicio de transporte, suponen la realización del hecho imponible del art. 4.1 de la Ley 27/2014 del Impuesto sobre Sociedades (en adelante, LIS). Los beneficios de las plataformas proceden, con carácter general, del cobro de comisiones por el servicio de intermediación, de la comercialización de la información suministrada por los usuarios o de la publicidad insertada en las citadas plataformas. De este modo, si consideramos que la titularidad de la misma corresponde a una persona jurídica, tributaría de acuerdo con lo dispuesto en el IS, mientras que si la plataforma fuese titularidad de una persona física, las rentas se calificarían como rendimientos de actividades económicas en el IRPF.

El problema surge cuando la persona jurídica titular de la plataforma no reside ni cuenta con una filial constituida en territorio español, ya que no serán contribuyentes del IS ex art. 7.1 LIS. Esto significa que, en principio, las entidades no residentes serían contribuyentes del IRNR, sin perjuicio de lo dispuesto en los Convenios de Doble Imposición Internacional (en adelante, CDI) conforme al art. 4 de la Ley 5/2004 del Impuesto sobre la 
Renta de no Residentes. En este sentido, el art. 7 del Modelo Convenio OCDE (en adelante, MCOCDE), relativo a los beneficios empresariales, establece un criterio de tributación compartida, condicionada a que la entidad opere mediante un establecimiento permanente en España. En el caso concreto hemos de atender al CDI entre los dos Estados implicados. En este punto, aparece el problema asociado a la indeterminación del «lugar fijo de negocios» desde el que operan las plataformas, y que conlleva la erosión de la base imponible. La Comisión Europea, consciente de esta cuestión, el 21 de marzo de 2018 ha propuesto nuevas reglas para garantizar un level playing field («reglas de juego uniformes») para todas las empresas, ya que las normas actualmente vigentes no permiten a los Estados Miembros gravar a las entidades digitales cuando no tienen una presencia física en los mismos. Este problema se abordará con una mayor concreción al hablar de las plataformas dedicadas al sector del alojamiento.

\section{La tributación en el sector del alojamiento}

El sector del alojamiento es uno de los ámbitos en los que la economía colaborativa ha irrumpido con mayor fuerza, pues en los últimos años es notable la creación de diversas plataformas digitales dedicadas a este sector, principalmente, para fines turísticos (v.gr. Airbnb, Wimdu, 9flats, Gloveler...). Estas plataformas sirven de punto de encuentro entre aquellos sujetos que ofrecen alquilar un bien inmueble (en su totalidad o parcialmente) durante un corto espacio de tiempo, y aquellos otros usuarios que buscan un alojamiento a un coste más reducido que el que supondría el establecimiento de hostelería. A diferencia del sector del transporte, en este caso, actualmente únicamente encontramos plataformas que se definen por prestar un servicio de intermediación, es decir, no prestan el servicio principal (alojamiento).

En principio, las relaciones que surjan en estas plataformas implicarán a particulares, pero no podemos descartar la posibilidad de que participen en ellas empresarios o profesionales. Así, por lo que se refiere al sujeto prestador del servicio (arrendador), en función de la calificación que merezca y de las circunstancias o condiciones en las que se desarrolle la actividad, podrá obtener rendimientos susceptibles de gravamen en el IRPF (rendimientos del capital inmobiliario, rendimientos del capital mobiliario, rendimientos de actividades económicas), o bien rendimientos gravados en el IS si se trata de una persona jurídica. Incluso, tratándose de un no residente, cabría hablar del IRNR. En cuanto a los otros dos sujetos intervinientes, el usuario (arrendatario o huésped) no percibirá ningún rendimiento susceptible de ser gravado, pues tan solo recibirá el servicio de alojamiento. Mientras que la plataforma, como persona jurídica, tributaría por el IS, salvo que se trate de un no residente, en cuyo caso, tributará por el IRNR según lo que disponga el CDI aplicable. 


\section{La tributación de las rentas percibidas por el prestador del servicio}

Conforme al art. 22.1 LIRPF, los rendimientos que el prestador del servicio de alojamiento obtenga como consecuencia de arrendar la totalidad o parte de un bien inmueble de su titularidad, se califican como rendimientos del capital inmobiliario (si se trata de un subarrendamiento se tratará de rendimientos del capital mobiliario ex art. 25.1 LIRPF). En consecuencia, tendrá derecho a deducir del rendimiento íntegro, de acuerdo con el art. 23.1 a) y b) LIRPF, todos los gastos necesarios para la obtención de los rendimientos, así como las cantidades destinadas a la amortización del inmueble. Respecto de los primeros (apartado a), dado que la lista de gastos deducibles contenidos en la ley es ejemplificativa, entendemos que cabría incluir las comisiones pagadas por el arrendador a la plataforma por su servicio de intermediación. Ahora bien, solo podrá deducirse el conjunto de los gastos si el arrendamiento abarca la totalidad del inmueble, pues en caso contrario, la deducción únicamente podría realizarse de manera proporcional a la parte del inmueble destinada a arrendamiento.

En relación con el arrendamiento parcial de la vivienda, si el inmueble constituye la vivienda habitual del prestador del servicio, es decir, de forma simultánea la utiliza como residencia y la destina al alquiler, nos podemos plantear qué sucede en ese caso con la deducción por inversión en vivienda habitual de la Disposición Transitoria 18. ${ }^{a}$ de la LIRPF. De acuerdo con la doctrina de la Dirección General de Tributos (en adelante, DGT), en su consulta de diciembre de $2015^{25}$, el arrendador puede seguir aplicando la deducción, pero solo en relación con la parte de la vivienda que utilice de forma privada, incluyendo las zonas comunes. Por tanto, se excluyen de la deducción aquellas zonas que se establezcan de uso reservado para el arrendatario con el que comparte la vivienda ${ }^{26}$.

En el supuesto de que el prestador del servicio sea una persona no residente en España que arrienda por medio de la plataforma un bien inmueble situado en territorio nacional, de acuerdo con el art. 6 del MCOCDE («rentas inmobiliarias»), las rentas obtenidas pueden ser sometidas a imposición en el Estado de la fuente. Se trata de una norma de tributación compartida, que, en el caso concreto, atribuirá a España la potestad de gravar dichas rentas a través del IRNR.

Por su parte, las rentas que el prestador del servicio obtenga como consecuencia del arrendamiento del bien inmueble podrían ser calificadas en virtud del art. 27 LIRPF como rendimientos de actividades económicas. En concreto, conforme a su apartado segundo, si el prestador del servicio tiene empleada a una persona con contrato laboral y a jornada completa, la actividad generará rendimientos de actividades económicas. Por lo tanto, esta

\footnotetext{
25 Consulta vinculante de la DGT, V3860-15.

26 En este sentido, puede verse SANZ GÓMEZ, R., «Airbnb, ¿economía colaborativa o economía sumergida? Reflexiones sobre el papel de las plataformas de intermediación en la aplicación de los tributos», ob.cit., p. 74, y ZAPATERO GASCO, A., «La tributación en el IRPF de los rendimientos percibidos a través de la plataforma Airbnb: aspectos controvertidos», Documento de Trabajo IEF, núm. 15, 2017, pp. 96-97.
} 
circunstancia permitirá calificar los rendimientos bajo una u otra categoría de renta. En este sentido, la DGT señala que, en el caso de alquileres de viviendas de uso turístico, si el servicio no solo se limita a la puesta a disposición de los inmuebles, sino que se complementa con la prestación de servicios propios de la industria hotelera, tales como restaurante, limpieza $^{27}$, lavado de ropa y otros análogos, las rentas tendrán la calificación de rendimientos de actividades económicas ${ }^{28}$. Por tanto, si no se prestan tales servicios nos encontraríamos ante rendimientos del capital inmobiliario, salvo que concurra la circunstancia del art. 27.2 LIRPF.

Como consecuencia de lo anterior, si se considera que el prestador del servicio es un profesional, el mismo estará sujeto al Impuesto sobre Actividades Económicas (IAE). No obstante, en virtud del art. 82.1 c) de la Ley Reguladora de las Haciendas Locales, las personas físicas están exentas, aunque habrán de presentar la declaración censal de alta. Así, si la actividad del contribuyente consiste en el arrendamiento sin prestación de servicios adicionales, habrá de darse de alta en el epígrafe 861.1 «alquiler de viviendas», mientras que si el alquiler se realiza con la prestación de servicios de hospedaje, en el grupo 685 «alojamientos turísticos extrahoteleros» ${ }^{29}$.

Todo lo referido anteriormente resulta de aplicación a prestaciones de servicios que encajan en el segundo modelo de negocio (v.gr. Airbnb, Wimdu, Homeaway). Sin embargo, en el caso de las plataformas propias del primer modelo de negocio, como, por ejemplo, Lovehomeswap o Guest to Guest, la calificación puede resultar distinta. Se trata de plataformas que ofrecen un servicio de intermediación en el intercambio de casas. En principio, y en atención a la clasificación realizada al inicio de este trabajo, podríamos considerar este tipo de operaciones como no sujetas a tributación por tratarse de una mera compartición de gastos. No obstante, de acuerdo con la definición legal de rentas en especie del art. 42 LIRPF, no podemos descartar la posible existencia de un contrato de arrendamiento con contraprestaciones en especie.

\section{La tributación de las rentas obtenidas por la plataforma}

Al igual que en el sector del transporte, la fiscalidad de las rentas obtenidas por la plataforma, en principio, no presenta dificultades, ya que los rendimientos obtenidos por el ejercicio de su actividad económica suponen la realización del hecho imponible del art.

\footnotetext{
27 No se consideran servicios complementarios propios de la industria hotelera, el servicio de limpieza y de cambio de ropa del apartamento a la entrada y salida del periodo contratado por cada arrendatario, el servicio de limpieza de las zonas comunes del edificio, así como los servicios de asistencia técnica y mantenimiento para eventuales reparaciones de fontanería, electricidad, cristalería... (Consulta vinculante de la DGT V2588-16).

28 Consultas vinculantes de la DGT, V2588-16 y V0731-17.

29 El IAE se encuentra regulado en el Real Decreto Legislativo 2/2004, de 5 de marzo, por el que se aprueba el texto refundido de la Ley Reguladora de las Haciendas Locales y en el Real Decreto Legislativo 1175/1990, de 28 de septiembre, por el que se aprueban las tarifas y la instrucción del impuesto sobre actividades económicas.
} 
4.1 LIS. La dificultad surge cuando la persona jurídica titular de la plataforma no reside ni cuenta con una filial constituida en territorio español, ya que no serán contribuyentes del IS ex art. 7.1 LIS. En este caso, las entidades no residentes podrían ser contribuyentes del IRNR. El ejemplo paradigmático en el sector del alojamiento es el de Airbnb.

La estructura empresarial de Airbnb está formada por una sede central que opera y está situada en Estados Unidos (Airbnb, Inc.); dos sociedades específicas que operan en la República Popular China y en Japón, respectivamente; y la gestión del resto de dominios web la lleva a cabo la filial irlandesa Airbnb Ireland UC. Lo cierto es que existe una filial española (Airbnb Marketing Services S.L.U) cuya actividad consiste en la mediación o marketing, si bien, conforme a la prueba practicada por el Juzgado de lo Contencioso-Administrativo de Barcelona, se considera que es «una sociedad no establecida en España la prestadora del servicio de la página web» ${ }^{30}$. Por último, interviene en la operación, una financiera del grupo como intermediadora en los pagos (Aibnb Payments UK Ltd).

De acuerdo con esta estructura empresarial, los rendimientos son obtenidos por una entidad no residente en España, en concreto, residente en Irlanda. De este modo, y en el mismo sentido que el MCOCDE, el CDI España-Irlanda establece en su art. 7 una norma de tributación compartida, condicionada a que la empresa realice su actividad en el Estado de la fuente por medio de un establecimiento permanente situado en él. En este punto, surge el problema asociado a la escasa o inexistente presencia física de las plataformas que se dedican a actividades digitales.

La economía digital está poniendo en entredicho el principio de que los beneficios han de ser gravados donde el valor es creado, pues las actuales normas tributarias fueron pensadas para negocios de «brick and mortar». Es decir, no incluyen a aquellos modelos de negocio en los que las entidades pueden ofrecer servicios digitales en un país, sin necesidad de estar allí presentes físicamente. Además, tampoco contemplan el papel de creación de valor que desempeñan los usuarios al proporcionar un contenido relevante como lo son los datos personales. Todo ello supone una desconexión entre el lugar en el que se crea el valor y el lugar en el que los tributos son pagados. En consecuencia, las entidades digitales se ven gravadas en menor medida, un $9.5 \%$ frente a un $23.2 \%$ de las entidades tradicionales ${ }^{31}$.

Tanto la OCDE como la UE, e incluso el Reino Unido, se han posicionado al respecto, si bien la OCDE y el Reino Unido de una manera más prudente ${ }^{32}$. En algunas

30 El fragmento entrecomillado procede de la Sentencia del Juzgado de lo Contencioso-Administrativo núm. 309/2016, de 29 de noviembre, y el resto de información sobre la estructura empresarial de Airbnb se encuentra disponible en la página web de la plataforma.

31 Communication from the Commission 21/03/2018: «Time to stablish a modern, fair and efficient taxation standard for the digital economy», p. 4.

32 La OCDE comenzó los trabajos en materia de economía digital en 2013 a través del grupo especial dependiente del Comité de Asuntos Fiscales. Los resultados de este grupo se publicaron en el Informe Final de la Acción 1 del Proyecto BEPS «Abordar los retos de la economía digital para la imposición». Recientemente, ha publicado el informe «Tax Challenges Arising from Digitalisation - Interim Report 2018». Por su parte, el 
jurisdicciones, ya se han implantado medidas en relación con la economía digital. Por ejemplo, en la India se estableció en el año 2016 un gravamen compensatorio sobre los servicios pagados por los anunciantes a entidades sin establecimiento permanente (equalisation levy).

La UE ha propuesto recientemente -el 21 de marzo de 2018- dos soluciones. La primera de ellas se prevé como una solución a corto plazo, que sería aplicable hasta alcanzar una solución a nivel internacional. Para tal fin, la Comisión contempla en su propuesta de Directiva la creación de un impuesto sobre los servicios digitales (Digital Services Tax) ${ }^{33}$, aplicable a un tipo de gravamen del $3 \%$ sobre los ingresos brutos anuales obtenidos en aquellos Estados Miembros en los que se encuentren los usuarios implicados. Este impuesto gravaría los ingresos procedentes de tres tipos de servicios relativos a la intermediación entre usuarios realizada por plataformas digitales; la transmisión de información sobre y generada por los usuarios; y, la colocación de publicidad dirigida a los usuarios integrantes de una interfaz (taxable revenues). Además, para la imposición del citado gravamen, tales servicios han de ser proporcionados por negocios con un total de más de 750.000.000€ anuales a nivel mundial, y, además, un total de más de 50.000.000€ anuales procedentes de actividades digitales desarrolladas en la UE (taxable person). Con la introducción de este impuesto, la UE estima que sus ingresos incrementarán en cinco billones de euros anuales. Esta propuesta ha sido calificada por algunos autores como «defectuosa», pues entre otros motivos, el establecimiento de los dos umbrales de ingresos referidos supone que el impuesto recae principalmente en empresas multinacionales estadounidenses, y solo en unas pocas entidades europeas ${ }^{34}$.

La segunda propuesta de la UE, prevista para un largo plazo, se basa en la denominada «presencia económica significativa» $\mathrm{o}$ «significant digital presence». La Directiva propone que los negocios digitales paguen impuestos en el Estado Miembro en el que tengan una presencia digital significativa, incluso aunque no tengan un establecimiento físico en el mismo. Para determinar tal presencia, se tendrán en cuenta tres umbrales o condiciones, teniendo en cuenta que basta con que se cumpla uno de ellos para considerar que existe un «establecimiento permanente virtual». Los criterios son los siguientes: que los ingresos procedentes de la prestación de servicios digitales a los usuarios de una jurisdicción excedan de 7.000.000€ en un periodo impositivo; que el número de usuarios en un Estado Miembro supere los 100.000 en un periodo impositivo; o que el número de contratos derivados de los

Reino Unido, ha mostrado su posición al respecto a través del «Corporate tax and the digital economy: position paper update» publicada en marzo de 2018 por la HM Treasury.

33 Proposal for a Council Directive on the common system of a digital services taxes on revenues resulting from the provision of certain digital services, 21/03/2018, pp. 24-25.

34 BECKER, J. y Englisch J., «EU Digital Services Tax: A Populist and Flawed Proposal», Kluwer International Tax Blog, entrada de blog de 16/03/2018, p. 2. Disponible en <http://kluwertaxblog. com/2018/03/16/eu-digital-services-tax-populist-flawed-proposal/>. [Consultado el 15/08/2019]. 
servicios digitales exceda los $3.000^{35}$. Estos criterios, desde nuestro punto de vista, también serían criticables por ser excesivamente objetivos, ya que no parecen tener en cuenta que el valor creado individualmente por los usuarios puede ser distinto en cada Estado con independencia del número de adscritos a una plataforma. Además, el hecho de que los requisitos sean alternativos y no acumulativos podría determinar que queden sujetas a una determinada jurisdicción entidades que realmente no tienen una presencia digital significativa.

En cualquier caso, todas las cuestiones relativas a la economía digital están aún por concretar, pues la apreciación de este problema así como las propuestas de solución, son muy recientes, si bien, a los efectos de este trabajo revela la íntima conexión que existe entre la economía colaborativa y la economía digital.

Para concluir este apartado relativo a la fiscalidad directa de las plataformas, hemos de hacer mención a una cuestión de especial relevancia y que puede ser de gran ayuda para la determinación de los ingresos que han de ser sujetos a imposición en el seno de la economía colaborativa. Nos estamos refiriendo a la colaboración de la plataforma con la Administración Tributaria a los efectos de proporcionarle la información necesaria para identificar a los beneficiarios de tales ingresos y así garantizar la aplicación de la normativa. De hecho, en gran medida, la aparición de un tercer sujeto en el consumo colaborativo tradicional puede ofrecer una ventaja para el control de operaciones que antes, probablemente, habrían formado parte de la «economía sumergida». Para ello, es necesaria la colaboración de las plataformas, que de ese modo, quedarían sujetas al cumplimiento de determinadas obligaciones formales ante la Administración Tributaria.

En este sentido, la plataforma Airbnb es una de las más implicadas en promover el cumplimiento tributario por parte de sus usuarios, pues en las condiciones de su página web, les informa de que «la normativa fiscal podrá requerir que recopilemos la debida información fiscal de nuestros anfitriones, o que retengamos impuestos de los pagos a nuestros anfitriones, o ambas cosas». De hecho, ya se puede afirmar que plataformas como Airbnb, han adquirido una obligación de información con la Agencia Tributaria establecida por el Real Decreto 1070/2017, de 29 de diciembre. Así, en su Exposición de Motivos se establece una nueva obligación de información «con fines de prevención del fraude fiscal para las personas o entidades, en particular, las denominadas "plataformas colaborativas", que intermedien en la cesión de uso de viviendas con fines turísticos». La Orden HFP/544/2018, de 24 de mayo, por la que se aprueba el modelo 179, «declaración informativa trimestral de la cesión de uso de viviendas con fines turísticos», establece las condiciones y el procedimiento para el cumplimiento de tal obligación de información. Con la finalidad de otorgar un plazo de adaptación, la citada Orden prevé que, exclusivamente para el ejercicio 2018, la declaración informativa tenga carácter anual, y que su plazo de presentación sea el comprendido entre el 1 y el 31 de enero de 2019. A partir de 2019, la periodicidad de la declaración será

Proposal for a Council Directive laying down rules relating to the corporate taxation of a significant digital presence, 21/03/2018, p. 16.

RJUAM, n. ${ }^{\circ}$ 39, 2019-I, pp. 139-161

ISSN: 1575-720-X 
trimestral, y deberá presentarse en relación con las operaciones realizadas en cada trimestre natural, en el plazo comprendido entre el primer y el último día del mes natural posterior a la finalización del trimestre a que se refiera la declaración ${ }^{36}$.

\section{FISCALIDAD INDIRECTA}

Antes de abordar el estudio del apartado relativo a la fiscalidad indirecta hemos de puntualizar que en el mismo, se tratará principalmente el Impuesto sobre el Valor Añadido (IVA) como consecuencia de las prestaciones de servicios que se originan en el seno de la economía colaborativa. Si bien en el apartado anterior se quiso diferenciar claramente entre el sector del alojamiento y el transporte, en este caso, el apartado se ha estructurado a partir de los posibles sujetos pasivos de este impuesto, pues la concreta categoría del servicio no genera muchas variaciones.

\section{La tributación del prestador del servicio}

De acuerdo con el art. 4 de la Ley 37/1992 del Impuesto sobre el Valor Añadido (en adelante, LIVA), estarán sujetas al citado tributo las entregas de bienes y prestaciones de servicios realizadas por empresarios o profesionales a título oneroso en el ámbito espacial del impuesto, con carácter habitual u ocasional, en el desarrollo de su actividad empresarial o profesional. Así, conforme al art. 5.1 c) LIVA se considera que los arrendadores de bienes son empresarios o profesionales y por tanto el arrendamiento de un bien inmueble estará sujeto al impuesto (concretamente, el arrendamiento se considera una prestación de servicios ex art. 11.2.2. ${ }^{\circ}$ LIVA).

Ahora bien, el art. 20.1. 23. ${ }^{\circ}$ LIVA establece una exención en el caso de que el arrendamiento se destine exclusivamente al uso de vivienda. De este modo, el arrendamiento de un inmueble, cuando se destine a tal fin, estará sujeto y exento a IVA ${ }^{37}$, teniendo en cuenta que quedan exceptuados los «arrendamientos de apartamentos o viviendas amueblados cuando el arrendador se obligue a la prestación de alguno de los servicios complementarios propios de la industria hotelera, tales como los de restaurante, limpieza, lavado de ropa u otros análogos». Esta cuestión es clave para determinar la exención o no al impuesto. En este

\footnotetext{
36 Para un análisis de la obligación de información, véase entre otros: SÁNCHEZ HUETE, M.A., «Las nuevas obligaciones informativas en la cesión de uso de viviendas con fines turísticos», en PEDREIRA MENÉNDEZ, J. (dir.), Fiscalidad de la colaboración social, (Aranzadi), 2018; MARTÍNEZ-CARRASCO PIGNATELLI, J.M., «Comentarios a la declaración informativa sobre alquileres turísticos (modelo 179)» Revista Quincena Fiscal, núm. 17, 2018; y, CALVO VÉRGEZ, J., «La obligación de información específica prevista en el ámbito tributario para las llamadas "plataformas colaborativas" que intermedien en la cesión del uso de viviendas con fines turísticos», Revista de contabilidad y tributación, núm. 434, 2019.

37 Téngase en cuenta que esta exención (limitada) no otorga al sujeto que realiza tales operaciones el derecho a deducir las cuotas de IVA que haya podido soportar.
} 
sentido, de acuerdo con la doctrina de la DGT no se consideran servicios complementarios propios de la industria hotelera, los servicios de limpieza y cambio de ropa en el apartamento prestado a la entrada y salida del periodo contratado por cada arrendatario; el servicio de limpieza de las zonas comunes así como de la urbanización en que esté situado; ni los servicios de asistencia técnica y mantenimiento para eventuales reparaciones de fontanería, electricidad, cerrajería y electrodomésticos ${ }^{38}$.

En definitiva, si el arrendamiento se destina al uso exclusivo de vivienda y no se prestan servicios complementarios, la actividad estará sujeta y exenta a IVA, sin perjuicio de que en virtud del art. 7.5 del Real Decreto Legislativo 1/1993, por el que se aprueba el Texto refundido de la Ley del Impuesto sobre Transmisiones Patrimoniales y Actos Jurídicos Documentados, esté sujeta a $\mathrm{TPO}^{39}$. Ahora bien, se ha de tener en cuenta que el citado Texto refundido ha sido recientemente modificado por el Real Decreto-Ley 7/2019, de 1 de marzo, de medidas urgentes en materia de vivienda y alquiler, de modo que los arrendamientos de vivienda para uso estable y permanente están exentos de TPO.

En el resto de casos, dicho arrendamiento estará sujeto y no exento de IVA, y por tanto, no sujeto a TPO. En concreto, será así cuando se alquile a personas jurídicas (pues no lo pueden destinar directamente a viviendas), o se presten servicios propios de la industria hotelera, o los arrendamientos de viviendas que sean utilizadas por el arrendatario para otros usos, tales como oficinas o despachos profesionales, etc.

Todo lo anterior se refiere al sector del alojamiento, pero igualmente en el sector del transporte el empresario o profesional que preste un servicio de este tipo será sujeto pasivo del IVA, pues conforme al art. 11.2.8. ${ }^{\circ}$ LIVA «los transportes» se consideran prestación de servicios a los efectos del impuesto.

Por último, se entenderá que el hecho imponible se ha realizado en el territorio de aplicación del impuesto cuando conforme al art. 69 LIVA, el destinatario del servicio sea un empresario con sede, establecimiento permanente, o domicilio en el territorio de aplicación del impuesto, o cuando el destinatario sea un particular y el prestador del servicio un empresario con sede o establecimiento permanente en el territorio de aplicación del impuesto. Ahora bien, se ha de tener en cuenta que en el caso del servicio de alojamiento se aplica la regla especial del art. 70.1.1. ${ }^{\circ}$ a) LIVA, por la cual se entenderá prestado el servicio en el territorio de aplicación del impuesto, si el mismo consiste en el arrendamiento o cesión de uso de un bien inmueble situado en tal territorio. Igualmente, también se considerará así para el servicio de transporte de pasajeros por la parte de trayecto que discurra por el territorio de aplicación del impuesto ex art.70.1.2. ${ }^{\circ}$ a) LIVA.

\footnotetext{
38 Consultas vinculantes de la DGT, V3095-14 y V0949-17.

39 A tenor de dicho precepto, no está sujeto al gravamen por TPO el arrendamiento de bienes inmuebles realizado por empresarios o profesionales en el ejercicio de su actividad económica y, en cualquier caso, cuando dicho arrendamiento esté sujeto a IVA. En el caso de que el arrendamiento de bienes inmuebles esté exento en el IVA, quedará sujeto al citado gravamen (salvo que el sujeto opte por renunciar a la exención).
} 


\section{La tributación de la plataforma}

Las plataformas intervienen en la economía colaborativa conectando al prestador del servicio con los usuarios y facilitan las transacciones entre ellos, de tal manera que prestan un servicio de intermediación por el que cobran comisiones (salvo en Uber, que como se indicó anteriormente, también presta el servicio de transporte). Esto supone que realizan el hecho imponible del IVA por prestar un servicio de mediación ex art. 11.2.15. ${ }^{\circ}$ LIVA, además de un servicio de transporte en el caso de Uber.

En cuanto a la localización de la prestación del servicio de mediación por las plataformas, pueden darse distintas situaciones en las que el hecho imponible se considera realizado en el territorio de aplicación del IVA. Por un lado, si la plataforma prestadora del servicio de mediación (empresario) tiene sede o establecimiento permanente en el territorio de aplicación del impuesto y el destinario del servicio es un particular, en virtud del art. 69.1.2. ${ }^{\circ}$ LIVA el hecho imponible se considera realizado en el territorio de aplicación del impuesto.

Por otro lado, se entenderá que el servicio ha sido prestado en el territorio de aplicación del impuesto cuando el destinatario sea un empresario o profesional con sede o establecimiento permanente o domicilio en el territorio de aplicación del impuesto, conforme al art. 69.1.1. ${ }^{\circ}$ LIVA. En este supuesto, si la plataforma prestadora del servicio de mediación no está establecida en el territorio de aplicación del impuesto, el empresario o profesional que reciba el servicio será sujeto pasivo por el mecanismo de inversión ex art. 84.1.2. ${ }^{\circ}$ a) LIVA, por lo que pagará a la plataforma el precio de la comisión sin IVA e ingresará la correspondiente cuota directamente a la Hacienda Pública («IVA autorrepercutido»).

No obstante lo anterior, en la prestación del servicio de mediación relacionado con bienes inmuebles, la regla especial del art. 70.1.1. ${ }^{\circ} \mathrm{d}$ ) LIVA permite considerar realizado el hecho imponible en el territorio de aplicación del impuesto, siempre y cuando el inmueble relacionado con el servicio radique en el mismo ${ }^{40}$. Por el contrario, los servicios de mediación prestados respecto del arrendamiento de inmuebles no situados en el territorio de aplicación del impuesto no estarán sujetos al IVA. Ahora bien, en este punto se ha de tener en cuenta que si el inmueble se encuentra en Canarias, Ceuta o Melilla, la operación no estará sujeta en el territorio de aplicación del IVA español, sin perjuicio de su sujeción al IGIC en Canarias de acuerdo con la Ley 20/1991 o al IPSI en Ceuta y Melilla según la Ley 8/1991.

Una vez que se ha determinado que el hecho imponible se ha realizado en el territorio de aplicación del impuesto conforme a la regla especial, se han de distinguir dos supuestos en el caso de que la plataforma no tenga sede o establecimiento permanente en el territorio de aplicación del impuesto, como es el caso de Airbnb. En primer lugar, cuando el destinatario del servicio sea un empresario o profesional, será este último el sujeto pasivo por el

\footnotetext{
40 Se entiende que el servicio de mediación es subsumible en el servicio de «gestión relativos a bienes inmuebles u operaciones inmobiliarias» al que se refiere este precepto.
} 
mecanismo de inversión comentado anteriormente ${ }^{41}$. En segundo lugar, si el destinatario del servicio es un particular, la prestadora del servicio de mediación habría de obtener un número de identificación fiscal español e incluir el IVA en la factura emitida.

Por lo que se refiere a la localización de la prestación del servicio de transporte realizado por Uber, el hecho imponible se entenderá realizado en el territorio de aplicación del impuesto conforme a la regla especial del art.70.1.2. ${ }^{\circ}$ a) LIVA.

\section{CONCLUSIONES}

El desarrollo de las nuevas tecnologías ha favorecido la irrupción de nuevos modelos de negocio que, a través de plataformas digitales, han incrementado los supuestos de consumo colaborativo hasta el punto de que ya podemos hablar de una economía colaborativa. Las ventajas de esta nueva realidad son claras para el panorama actual de crisis económica, pues además de promover un consumo más eficiente, favorece el emprendimiento profesional y con ello la creación de empleo. No obstante, el vacío legal en relación con los derechos y deberes de los sujetos intervinientes en esta nueva realidad, hace necesario un esfuerzo regulatorio de los Estados que aporte seguridad jurídica e impulse estos nuevos modelos de negocio.

El principal problema que ha de abordarse es la propia delimitación del concepto de economía colaborativa, y con ello la calificación de los modelos de negocio y sujetos intervinientes en la misma. Ello se reflejará en el tratamiento tributario que merezcan las posibles rentas obtenidas por los distintos actores, así como en la resolución del resto de cuestiones que se plantean en otras ramas del Derecho. Es por ello que hemos propuesto la clasificación de las plataformas en tres modelos de negocio perfectamente diferenciables, a la espera de una solución a nivel de la UE.

En este sentido, es posible diferenciar un primer modelo de negocio denominado de «filosofía colaborativa» en el que la finalidad principal perseguida por los usuarios es la compartición de gastos; un segundo modelo en el que existe un ánimo de obtener ingresos adicionales mediante el aprovechamiento de bienes infrautilizados; $\mathrm{y}$, un tercer modelo de negocio, de dudosa adscripción a la economía colaborativa, en el que la plataforma no solo se limita a prestar un servicio de intermediación sino que presta el servicio subyacente.

Partiendo de la anterior distinción, el sometimiento a gravamen de las posibles rentas procedentes de la compartición de gastos puede ser discutible si en determinados casos se obtienen ingresos ocasionales, por lo que resulta conveniente valorar el establecimiento de

\footnotetext{
41 En este caso, el destinatario del servicio (empresario o profesional) deberá tener un número de operador intracomunitario (NIF-IVA) e informar la operación en el modelo 349 «Declaración recapitulativa de operaciones intracomunitarias», indicando el número de operador intracomunitario (VAT number) del prestador (por ejemplo, Airbnb).
} 
exenciones o supuestos de no sujeción hasta ciertos umbrales de renta. Por otro lado, en los casos en los que existe un claro ánimo de lucro, la calificación de los actores intervinientes, así como de las interacciones entre los mismos es determinante para la fiscalidad. En este sentido, la STJUE de 20 de diciembre de 2017 ha arrojado cierta claridad en lo que a Uber se refiere, y puede suponer un avance en la clasificación de muchas de las plataformas colaborativas actuales. De esta forma, la delimitación de las rentas en una concreta categoría no resulta una tarea difícil, teniendo en cuenta que no consideramos necesaria la creación de figuras impositivas específicas, sino que las existentes han de adaptarse a las nuevas realidades. Sin embargo, apreciamos que la principal problemática tributaria se encuentra asociada a las plataformas digitales.

La creciente evolución de la economía digital está quebrando el principio por el cual los beneficios han de ser gravados donde el valor es creado, pues la mínima o inexistente presencia física de estas entidades impide a las jurisdicciones la aplicación de las normas tributarias actuales. Por ello, urge una reforma de las normas internacionales relativas a los beneficios empresariales, en las que se tenga en cuenta como principal criterio el valor generado por los usuarios con su participación en estos modelos de negocio. Por el momento hemos de esperar a la resolución de las propuestas vigentes, si bien las iniciativas de la UE al respecto no parece que estén teniendo buena acogida.

Además, en relación con las plataformas, hemos de concluir que pueden desempeñar un papel fundamental en el control tributario y la sujeción a gravamen de las rentas generadas en el seno de la economía colaborativa. Así, resulta esencial la colaboración de las plataformas con la Administración tributaria en lo referente al suministro de información tributaria y en la recaudación, pudiendo incluso adoptar en ocasiones la posición jurídica de sustituto del contribuyente.

Por último, en lo que se refiere a la fiscalidad indirecta será fundamental determinar la localización de la prestación del servicio para así poder gravar los ingresos en el territorio de aplicación del impuesto. La tarea será sencilla en el sector del alojamiento, pues si el bien inmueble se encuentra situado en el territorio de aplicación del impuesto, los servicios relacionados con el mismo serán gravados en dicho territorio. Del mismo modo, sucede en el sector del transporte por la parte de recorrido que se realiza en dicho territorio.

\section{BIBLIOGRAFÍA}

ANTÓN ANTÓN, Á., «Implicaciones fiscales de la economía digital en la era digital», en MORENO GONZÁLEZ, S. (dir.), y GÓMEZ REQUENA, J.A. (ed.), Tendencias y desafíos fiscales de la economía digital, (Aranzadi), 2017.

ANTÓN ANTÓN, Á. y BILBAO ESTRADA, I., «El consumo colaborativo en la era digital: un nuevo reto para la fiscalidad», en Documento de Trabajo IEF, núm. 26, 2016, pp. 1-39. 
BECKER J. y Englisch, J., «EU Digital Services Tax: A Populist and Flawed Proposal», Kluwer International Tax Blog, 16/03/2018 [Consultado el 15/08/2019].

Bruselas, 2.6.2016 COM (2016) 356 final Comunicación de la Comisión al Parlamento Europeo, al Consejo, al Comité Económico y Social Europeo y al Comité de las Regiones: Una Agenda Europea para la economía colaborativa.

Brussels, 6 November 2017. Cover note from General Secretariat of the Council to Delegations.

Brussels, 2.6.2016 SWD (2016) 184 final Commission Staff Working Document, accompanying the document: Communication from the Commission to the European Parliament, the Council, the European Economic and Social Committee and the Committee of the Regions: A European Agenda for the collaborative economy - supporting analysis.

Communication from the Commission 21/03/2018: «Time to stablish a modern, fair and efficient taxation standard for the digital economy».

Dictamen del Comité Económico y Social Europeo sobre la «Comunicación de la Comisión al Parlamento Europeo, al Consejo, al Comité Económico y Social Europeo y al Comité de las Regiones - Una Agenda Europea para la economía colaborativa», 521. ${ }^{\circ}$ Pleno del CESE de los días 14 y 15 de diciembre de 2016.

HERNÁNDEZ BEJARANO, M., «El apoyo europeo al modelo de economía colaborativa: algunas cuestiones y propuestas para afrontar una regulación laboral y de Seguridad Social», en Revista Española de Derecho del Trabajo, núm. 192, 2016, pp. 165-186.

JARNE MUÑOZ, P., «El prosumidor como figura clave en el desarrollo del Derecho del Consumo derivado del mercado digital», en Revista CESCO de Derecho de Consumo, núm. 19, 2016, pp. 41-51.

LUCAS DURÁN, M., «Problemática jurídica de la economía colaborativa: especial referencia a la fiscalidad de las plataformas», en Anuario de Facultad de Derecho (Universidad de Alcalá), núm. 10, 2017, pp. 131-172.

MACHANCOSES GARCÍA, E., «Economía de plataforma en los servicios de transporte terrestre de pasajeros: Retos tributarios de la imposición directa sobre el usuario y la plataforma», en Revista Quincena Fiscal, núm. 15, 2017, pp. 41-82.

MARTÍNEZ MUÑOZ, Y., «El tratamiento fiscal del crowdfunding», en Revista Quincena Fiscal, núm. 14, 2015, pp. 59-86.

MONTESINOS OLTRA, S., «Los actores de la economía colaborativa desde el punto de vista del Derecho Tributario», en Revista Economía Industrial, núm. 402, 2016, pp. 47-54. 
Proposal for a Council Directive on the common system of a digital services taxes on revenues resulting from the provision of certain digital services, 21/03/2018.

Proposal for a Council Directive laying down rules relating to the corporate taxation of a significant digital presence, 21/03/2018.

SANZ GÓMEZ, R., «Airbnb, ¿economía colaborativa o economía sumergida? Reflexiones sobre el papel de las plataformas de intermediación en la aplicación de los tributos», en Documento de Trabajo IEF, núm. 15, 2017, pp. 64-83.

ZAPATERO GASCO, A., «La tributación en el IRPF de los rendimientos percibidos a través de la plataforma Airbnb: aspectos controvertidos», Documento de Trabajo $I E F$, núm. 15, 2017, pp. 84-107. 